\title{
Atención post hospitalaria de pacientes con accidente cerebrovascular en atención primaria de salud
}

\author{
Carolina E. Leyton Pavez Doctorada en Dirección y Administración de Empresas y Magister en Gestión de Instituciones de Salud. Académica del \\ Departamento de Gestión Empresarial, Facultad de Ciencias Empresariales, Universidad del Bío-Bío. Chillán, Chile. \\ Grupo Investigación Gestión en Salud, Facultad de Ciencias Empresariales, Universidad del Bío-Bío. Chillán, Chile. cleyton@ubiobio.cl \\ Iván R. Paul Espinoza. Magíster y Médico Ginecólogo, Director de la Dirección Servicio de Salud Ñuble. Chillán, Chile. ivan.paul@redsalud.gov.cl \\ Grupo Investigación Gestión en Salud, Facultad de Ciencias Empresariales, Universidad del Bío-Bío. Chillán, Chile. \\ Priscila A. Hernández Poblete Ingeniero en Administración de Empresas, Jefa de la Oficina de Gestión de Garantías en Salud del Centro de Salud Familiar \\ Violeta Parra. Chillán, Chile. priscila.hernandez@redsalud.gov.cl \\ Joan C. Gil Martín Doctor en Dirección y Administración de Empresas y Académico del Departamento de Organización de Empresas. \\ Universidad Politécnica de Cataluña. Barcelona, España. joan.carles.gil@upc.edu
}

Fecha de recepción: 05/12/2017

Fecha de corrección: 05/12/2018

Fecha de aprobación: 02/03/2019

Fecha de publicación: 30/06/2019

\begin{abstract}
Resumen
Introducción: el desarrollo constante de estrategias locales de prevención, promoción, acciones curativas y de rehabilitación para personas con Accidente Cerebrovascular, permite reducir la letalidad, discapacidad y complicaciones. OBJETIVO: elaborar un Plan de Intervención Integral para la atención post hospitalaria de pacientes con Accidente Cerebrovascular en un Centro de Salud Familiar chileno. MÉTODOS: para diseñar el plan fue necesario elaborar un diagnóstico situacional, identificar los factores de riesgo, y caracterizar la población durante el periodo del 20092013. Luego junto a directivos, referentes técnicos y Equipo de Salud Cardiovascular se identificaron las estrategias de educación, cuidado y tratamiento que conformaron el plan. Resultados: entre un $40 \%$ a un $50 \%$ de las personas con Accidente Cerebrovascular presentan secuelas que requieren algún grado de apoyo, es por ello que el Plan de Intervención Integral integra diferentes profesionales de la salud, los que a través de terapias y técnicas colaboran para conseguir una situación funcional, familiar y social lo más próxima a la que el paciente tenía previo al accidente. CONCLUSIÓNES: un enfoque integral e interdisciplinario desde la atención primaria permite disminuir las complicaciones prevenibles, apoyar e integrar al paciente y a su familia en el proceso de tratamiento, disminuir el grado de discapacidad, optimizar los resultados funcionales y la reintegración social
\end{abstract}

Palabras Claves: Estrategia; Cerebrovascular; Atención Primaria.

Copyright (C) Facultad de Ciencias de la Salud de la Universidad Tecnológica de Pereira. 1995-2018. Todos los derechos reservados
Hospital post attention of patients with vascular brain accident in primary health care

\begin{abstract}
Introduction: The constant development of local strategies for prevention, promotion, curative and rehabilitation actions for people with Vascular Brain Accident, can reduce lethality, disability and complications.

Objective: to develop an Integral Intervention Plan for the posthospital care of patients with Vascular Brain Accident in a Chilean Family Health Center. METHODS: to design the plan it was necessary to develop a situational diagnosis, identify the risk factors, and characterize the population during the period 2009-2013. Next, along with managers, technical referents and cardiovascular health team, the education, care and treatment strategies that shaped the plan were identified.

Results: between $40 \%$ and $50 \%$ of people with Vascular Brain Accident have sequelae that require some degree of support, which is why the Integral Intervention Plan integrates different health professionals, who through therapies and techniques collaborate To achieve a functional, family and social situation as close as the patient had before the accident. CONCLUSIONS: a comprehensive and interdisciplinary approach from primary care allows reducing preventable complications, supporting and integrating the patient and his family in the treatment process, reducing the degree of disability, optimizing functional outcomes and social reintegration.
\end{abstract}

Key Words: Strategy; Vascular Brain; Primary Care.

Copyright (C) Facultad de Ciencias de la Salud de la Universidad Tecnológica de Pereira. 1995-2018. Todos los derechos reservados

\section{Introducción}

De acuerdo a la Organización Panamericana de la Salud (OPS) de la Organización Mundial de Salud (OMS) (1) las enfermedades cardiovasculares (ECV) son causadas por los trastornos del corazón y vasos sanguíneos. Dentro de este grupo se encuentra la cardiopatía coronaria (ataques cardíacos), enfermedad cerebrovascular (accidente cerebrovascular), hipertensión (presión arterial elevada), arteriopatía periférica, cardiopatía reumática, cardiopatía congénita e insuficiencia cardíaca. Y las principales causas son el consumo de tabaco, la inactividad física y un régimen alimentario insalubre. 
La OPS/OMS (1) calcula que en el año 2012 murieron por ECV: 17,5 millones de personas, lo cual representa un $31 \%$ de todas las muertes registradas en el mundo. De estas muertes, 7,4 millones se debieron a la cardiopatía coronaria, y 6,7 millones, a los accidente cerebrovascular (ACV). Por otra parte más del $80 \%$ de muertes tienen lugar en los países de ingresos bajos y medianos, ocurren casi por igual en los hombres y las mujeres y se estima que éstas continuarán siendo la principal causa de muerte a nivel mundial (2).

Las ECV corresponden a la principal causa de mortalidad en Chile, con más de 24 mil defunciones anuales, representando cerca de un tercio de todas las muertes. Respecto de la carga de enfermedad, las ECV se ubican en el tercer lugar, tanto en hombres como en mujeres, principalmente a expensas de generación de discapacidad (4).

En Chile las diferencias geográficas en la mortalidad por ECV también son un elemento importante a considerar, ya que tasas más bajas se presentan en el norte del país, Tarapacá y Atacama, y tasas significativamente mayores que el promedio nacional en las Regiones del Maule y Bio Bio (5). El MINSAL (6) se explica que las causas de la variabilidad podrían estar relacionadas con: diferencias en la prevalencia de los factores de riesgo cardiovascular, acceso y calidad de la atención de estas enfermedades y, también, diferencias socioeconómicas. Esta información es relevante en la formulación de estrategias y metas regionales.

Los ACV (7), corresponden a afecciones vasculares del encéfalo, que incluye los siguientes subtipos patológicos: enfermedades isquémicas $(65 \%)$, hemorragias intracerebrales $(23 \%)$ y hemorragias subaracnoideas $(5 \%)$. Esta distinción en subtipos patológicos es importante, porque el enfrentamiento puede variar tanto en su prevención y su manejo agudo, como en su pronóstico.

El ACV puede afectar diversos ámbitos, dejando secuelas como parálisis, déficit en la coordinación motora, déficits cognoscitivos, problemas del habla, dificultades emocionales dolor y trastornos sensitivos, los que pueden generar problemas en las actividades de la vida diaria (8). Entre las principales se encuentran:

- Déficits cognoscitivos: problemas de raciocinio, conciencia, atención, aprendizaje, juicio y memoria. Si los problemas son más severos, el paciente puede presentar por ejemplo una apraxia, agnosia y negligencia (el paciente no tiene conocimiento de un lado del cuerpo o la visión).

- Déficits de habla y lenguaje: generalmente se presenta como afasia (déficit de comprensión o emisión de lenguaje) y disartria (problemas al hablar). Los problemas de lenguaje resultan generalmente del daño a los lóbulos frontales y parietotemporales izquierdos.

- Déficits emocionales: pueden tener dificultad en controlar sus emociones o expresar emociones inapropiadas en ciertas situaciones. Estos pacientes generalmente sufren de depresión cuyo comportamiento clínico puede dificultar la recuperación y la rehabilitación e incluso puede concluir en un suicidio.

La OMS (9) por su parte indica que la disminución de la mortalidad cardiovascular en países desarrollados de debe a una mejor prevención, diagnóstico y tratamiento, junto a una menor prevalencia de tabaquismo, y menores niveles de presión arterial y colesterol total.

Según estudio realizado por la Unidad del Epidemiologia de la región del Bio Bio en Chile el año 2013 (10) señala que la tasa regional de mortalidad por ECV, es de 74 por cien mil (2010) es más alta que la nacional de 52 casos por cien mil. Dentro de la región del Bio Bio es la provincia de Ñuble la que supera a las demás provincias, con tasas de mortalidad elevadas durante todo el periodo, al inicio presenta tasas que variaban entre 90 muertes cada 100.000 habitantes (1990) hasta 101 muertes por cien mil (1993), en los últimos años estas tasas llegan a 76 por cien mil (2009) y 74 por cien mil (2010), 22 unidades sobre la tasa nacional. Además dicho estudio indica que de las 10 comunas con las más altas tasas de mortalidad por ACV en la región, 4 corresponden a la provincia de Nuble, y de las 7 comunas con las más altas tasas de mortalidad por ACV según nivel de instrucción bajo ( $<8$ años), 6 pertenecen a la provincia de Ñuble.

Para (11) la prevención y el control de las ECV son de los principales desafíos sanitarios del país. Esto requiere de un mayor compromiso (recursos) no solo del sector salud sino también de otros sectores, para reducir la prevalencia de los factores de riesgo cardiovasculares en la población. A nivel de la red de servicios de salud, es fundamental fortalecer y ampliar la oferta de servicios de salud preventivos en la atención primaria de salud y mejorar la articulación entre los distintos niveles de atención para asegurar la continuidad y una mejor calidad de la atención. Por lo que "En respuesta a la transición epidemiológica acelerada observada en Chile que se caracteriza por un incremento absoluto y relativo de la población adulta y de los mayores de 65 años, y el aumento en la prevalencia de las enfermedades no transmisibles y sus factores de riesgo, se han desarrollado programas de prevención y control para estas patologías en la atención primaria de salud". Entre otros, se destaca el Programa Salud Cardiovascular (PSCV), con más de un millón y medio de personas con diabetes, hipertensión, dislipidemia o tabaquismo en control".

El PSCV, a cargo de un Equipo Interdisciplinario (médico, enfermera, nutricionista y auxiliar paramédico al menos, y el apoyo de asistente social, psicólogo y kinesiólogo o profesor de educación física, según disponibilidad). Este "nace" el 2002 (12) producto de la reorientación de los subprogramas de Hipertensión Arterial (HTA) y Diabetes (DM), cuyo principal cambio fue incorporar el enfoque de riesgo cardiovascular (CV) global en el manejo de las personas bajo control, en lugar de tratar los factores de riesgo en forma separada, esto ha permitido mejorar la capacidad de diagnóstico, una mayor gama de opciones terapéuticas, asegurar el tratamiento medicamentoso e incrementar la dotación de recursos humanos y otras mejorías según las necesidades de los niveles locales. Adicionalmente, a contar del 2005, el manejo de la hipertensión, diabetes, infarto agudo al miocardio, se incorporan como Garantías Explícitas de Salud (GES), en tanto que el accidente cerebrovascular isquémico lo hace a partir de 2006., utiliza un enfoque terapéutico basado en el nivel de riesgo cardiovascular absoluto, cuyo objetivo es mejorar la eficiencia en el uso de los recursos y su efectividad. Las personas inscritas en el PSCV tienen derecho a las garantías explícitas de salud asociadas a la hipertensión y la diabetes, más otras prestaciones o servicios adicionales vinculados a la organización para el diagnóstico, tratamiento y seguimiento de los pacientes (13).

No obstante es primordial el desarrollo constante de estrategias (14) locales para la prevención, promoción y acciones curativas para los pacientes con factores de riesgos además de aquellos que han sufrido un $\mathrm{ACV}$, estableciendo planes y flujos eficientes para dar seguimiento y continuidad en la atención post hospitalaria a aquellos pacientes desde la atención primaria.

La presente investigación en primer lugar realiza un diagnóstico situacional de casos y población con ACV y sus factores de riesgo durante el periodo del 2009-2013, luego analiza la prevalencia e incidencia del ACV en el principal Centro de Salud Familiar Violeta Parra de la provincia de Ñuble en Chile, con la finalidad de identificar a estos pacientes y realizar intervenciones para mejorar su calidad de vida mediante un Plan de Intervención Integral.

Dicho Plan de Intervención Integral busca recuperar la independencia funcional e integración familiar, social y laboral, de acuerdo a las capacidades remanentes del paciente (motora, cognitiva y emocional), utilizar el máximo provecho del recurso humano especializado en la 
atención primaria, en el cual el paciente debe ser evaluado y manejado durante su atención post hospitalaria, por un Equipo Interdisciplinario, junto con el paciente y su familiar o cuidador, de acuerdo a sus necesidades. Es por ello que la presente investigación elabora un Plan de Intervención Integral para la atención post hospitalaria de pacientes con Accidente Cerebrovascular en un Centro de Salud Familiar chileno, para lo cual se realiza un diagnóstico situacional de casos de ACV y sus factores de riesgo en la provincia de Ñuble, se caracteriza la población con ACV del Centro de Salud Familiar durante el periodo del 2009-2013 y se identifican las estrategias efectivas de educación, cuidado y tratamiento para la atención post hospitalaria de pacientes con Accidente Cardiovascular.

\section{Métodos}

La etapa descriptiva permite aclarar y definir la real naturaleza del problema, sus tendencias, comportamientos. Para obtener esto de manera confiable, se utilizan principalmente, fuentes de información oficial, estudios con evidencias científicas y guías clínicas con orientación a la atención de aquellos pacientes que sufren un ACV en la provincia. Dicha información también reporta antecedentes de la eficacia del uso de intervenciones preventivas, diagnósticas, curativas y de rehabilitación, en el abordaje del ACV en la atención primaria de salud.

Para el análisis estadístico se construyó una base de datos a partir de los reportes extraídos del Sistema de Información para las Garantías Explicitas de Salud (SIGGES), sistema creado por el Fondo Nacional de Salud (FONASA) para el monitoreo de los problemas de Salud de las Garantías Explicitas de Salud (GES), de pacientes que cursaron ACV entre los periodos 2009-2013, analizando características tales como sexo y edad. Utilizando para ello la herramienta Excel, principalmente por su facial uso y capacidad que presenta para tratar datos, con el propósito de obtener mayor conocimiento del comportamiento de las variables y lograr las comparaciones y análisis necesarios.

Para la elaboración del Plan de Intervención Integral, se aplican técnicas tales como entrevistas en profundidad e instrumentos como cuestionarios a directivos, referentes técnicos y equipo de salud Cardiovascular del Centro de Salud Familiar. Una vez definidas las estrategias se procedió al costeo de dichas intervenciones.

\section{Resultados}

La incidencia de ACV, de acuerdo a la tabla 1 se observa que:

- En la provincia de Nuble el número más alto de eventos de ACV se presentó el año 2013, con 1.106 casos, y entre el periodo 2009 y 2013 existe un $26 \%$ de aumento de casos de ACV.

- $\quad$ El número de pacientes que cursaron ACV desde el año 2009 al 2013, siendo este el año 2012 con 125 casos el que presentó un mayor número de eventos. Se observa un aumento de un $43 \%$ entre el año 2009 y el 2012, lo que corresponde a 54 casos más que el año 2009.

- En cuanto al sexo, no se presenta una diferencia significativa, lo que se puede interpretar que durante el periodo no fue un factor de riesgo determinante, aunque en el año 2012 existe un predominio en el sexo femenino con 70 casos en comparación con 55 que fueron masculinos.

- La clasificación de pacientes que cursaron ACV según rango de edad, se tiene que desde los 65 años es donde se presentan la mayor cantidad de casos siendo esta edad uno de los mayores factores de riesgo.

- El grupo entre 46 a 64 años también presenta una cantidad importante de casos de ACV los cuales son pacientes en edad autovalentes por lo tanto son un grupo a los cuales se debe intervenir para prevenir un nuevo ACV.

Tabla 1: Casos de pacientes con ACV, durante el año 2009 al 2013.

\begin{tabular}{l|c|c|c|c|c|c}
\hline \multicolumn{7}{c}{ Casos ACV provincia de Nuble, las 21 comunas. } \\
\hline & 2009 & 2010 & 2011 & 2012 & 2013 & Total \\
\hline $\begin{array}{l}\text { Pacientes } \\
\text { con ACV }\end{array}$ & 982 & 738 & 930 & 914 & 1.106 & 4.670 \\
\hline \multicolumn{7}{c}{ Casos ACV Centro de Salud Familiar, según sexo. } \\
\hline Femenino & 35 & 43 & 38 & 70 & 51 & 237 \\
\hline Masculino & 36 & 47 & 39 & 55 & 46 & 223 \\
\hline Total & 71 & 90 & 77 & 125 & 97 & 460 \\
\hline \multicolumn{7}{|c|}{ Casos ACV Centro de Salud Familiar, según edad. } \\
\hline $20-35$ & 1 & 1 & 0 & 0 & 1 & 3 \\
\hline $36-45$ & 1 & 4 & 2 & 1 & 2 & 10 \\
\hline $46-64$ & 11 & 21 & 13 & 21 & 14 & 80 \\
65 y Mas & 58 & 64 & 62 & 103 & 80 & 367 \\
\hline Total & 71 & 90 & 77 & 125 & 97 & 460 \\
\hline
\end{tabular}

La prevalencia corresponde a la proporción de personas con ACV del total de inscritos en el Centro de Salud Familiar durante el 20092013. O la proporción de pacientes diagnosticados con ACV sobre la población inscrita durando el periodo estudiado.

En la tabla 2 se puede observar que:

- Las personas inscritas en el Centro de Salud Familiar desde el año 2009 al 2013 han aumentado en un 5\%.

- La cantidad de pacientes acumulados que cursaron un ACV pertenecientes al Centro de Salud Familiar desde el año 2009 al 2013, va desde 71 el año 2009 a 460 pacientes el año 2013.

Tabla 2: Prevalencia ACV Centro de Salud Familiar

\begin{tabular}{l|c|c|c|c|c}
\hline \multicolumn{1}{c|}{ Año } & 2009 & 2010 & 2011 & 2012 & 2013 \\
\hline $\begin{array}{l}\text { Población Ins- } \\
\text { crita }\end{array}$ & 62.130 & 63.110 & 64.250 & 65.102 & 65.536 \\
\hline $\begin{array}{l}\text { Eventos ACV } \\
\text { Acumulados }\end{array}$ & 71 & 161 & 238 & 363 & 460 \\
\hline Prevalencia ACV & $0,11 \%$ & $0,26 \%$ & $0,37 \%$ & $0,56 \%$ & $0,70 \%$ \\
\hline
\end{tabular}

Para la investigación de desarrollaron una serie de entrevistas a los referentes técnico y expertos del Servicio de Salud y del Centro de Salud Familiar, con el propósito de realizar un diagnóstico situacional del tema en estudio.

De acuerdo a la entrevista realizada a los expertos se abordaron preguntas clínicas para poder elaborar el Plan de Intervención Integral.

\section{Preguntas Abordadas:}

¿Cuál es el objetivo de la intervención post alta hospitalaria de los pacientes con $\mathrm{ACV}$ ?

- ¿Cuáles son los plazos de recuperación post ACV?

- ¿Cuándo iniciar la intervención? 
- ¿Quiénes deben formar parte del equipo de intervención integral?

- ¿Cómo organizar el plan de intervención del paciente post ACV en la Atención Primaria?

De acuerdo a los resultados de las entrevistas, se obtuvieron los siguientes resultados:

- Luego de ocurrido un ACV, la evolución más probable es de recuperación la que dependerá de cada paciente (raramente será el $100 \%)$.

- Los factores de riesgo más importantes son la diabetes, la hipertensión, el colesterol alto y también los antecedentes familiares directos.

- $\quad$ Entre un $40 \%$ a un $50 \%$ de las personas que sufren accidentes cerebrales tienen secuelas que van a requerir algún grado de apoyo. Por eso se debe intervenir con un equipo que a través de diversas terapias y técnicas colabora en la rehabilitación para conseguir una situación funcional, familiar y social lo más próxima a la que el paciente tenía previo al ACV.

- La intervención es un proceso que con enfoque integral e interdisciplinario debe buscar disminuir la incidencia de las complicaciones prevenibles, apoyar e integrar al paciente y a su familia en proceso de tratamiento, disminuir el grado de discapacidad, y optimizar los resultados funcionales y la reintegración social.

- Un Plan de Intervención Integral considera además, la identificación de factores biomédicos, psicológicos y sociales previos al ACV que pudieran incidir en la evolución del paciente. Los factores predictores más importantes en la recuperación funcional de la persona con ACV son la severidad del compromiso inicial y la edad; usando ambos parámetros sumando el análisis de los factores personales y ambientales se puede determinar las necesidades terapéuticas y recursos para su rehabilitación.

- Existe evidencia que la intervención y rehabilitación precoz e intensiva se asocia a una disminución de la morbimortalidad asociada y mejora el desenlace funcional.

- Los pacientes que hayan sufrido un $\mathrm{ACV}$, una vez contrarreferidos a su establecimiento de origen, deberán ser evaluados por el Equipo de Salud Cardiovascular para su ingreso al programa y/o reclasificación de riesgo.

- Toda intervención debe responder a un plan el que se inicia con una evaluación del Equipo Interdisciplinario centrado en las necesidades del paciente y tomando en consideración su entorno psicosocial, en este equipo se requiere la participación de un equipo organizado compuesto por médico, enfermera, nutricionista, kinesiólogo, fonoaudiólogo, psicólogo y apoyo de medicina complementaria. Además de derivaciones al nivel secundario con especialistas de acuerdo a las necesidades o complicaciones específicas del paciente.

- Los pacientes deben mantenerse bajo control, sobre todo aquellos con mayores factores de riesgos asociados ya que están expuestas a desarrollar un nuevo evento cerebrovascular.

- Para asegurar el mejor desenlace funcional posible, el equipo debe actuar de acuerdo a protocolos, guías de práctica clínica, procesos clínicos integrados sujetos a control de calidad y auditoría.
A continuación se presentaron las estrategias identificadas para el Plan de Intervención Integral cuya finalidad es proporcionar una atención integral de calidad, eficiente y eficaz, con el fin de reducir las consecuencias de la enfermedad y la dependencia en los pacientes, mediante la prestación de servicios adecuados en cada momento.

\section{Las terapias de rehabilitación post ACV se pueden dividir en:}

- Terapia Física: esta terapia en muchos pacientes se vuelve la piedra angular en el proceso de rehabilitación. Está a cargo de un kinesiólogo, el cual utiliza la enseñanza, los ejercicios y la manipulación física del cuerpo del paciente con la intención de restaurar el movimiento, equilibrio y la coordinación. El objetivo de la terapia física es lograr que el paciente que sufre un ACV, vuelva a aprender actividades motoras simples, tales como caminar, sentarse, ponerse de pie, acostarse y el proceso de cambiar de un tipo de movimiento a otro.

- Terapia ocupacional: los objetivos de esta terapia incluyen volver a aprender a comer, beber, vestirse, bañarse, cocinar y el cuidado personal.

- Terapia de dicción o del habla: Esta terapia está a cargo de un fonoaudiólogo, cuyo objetivo principal es enseñar el lenguaje y las destrezas de comunicación.

- Terapia psicológica. Esta terapia consiste en aliviar algunos problemas mentales y emocionales relacionados (depresión).

La intervención es un proceso que con enfoque integral e interdisciplinario busca disminuir la incidencia de las complicaciones prevenibles, apoyar e integrar al paciente y a su familia en proceso de tratamiento, disminuir el grado de discapacidad, y optimizar los resultados funcionales y la reintegración social.

\section{Intervención Seguimiento y Tratamiento:}

- La intervención se realizará al momento del alta hospitalaria, el Centro de Salud Familiar, es informado del evento cerebrovascular a través de un documento de contrarreferencia desde el hospital a la oficina GES.

- Este documento será enviado a coordinadores de sector del Centro de Salud Familiar, para intervenir y citar a estos pacientes para ser evaluados por el Equipo de salud Cardiovascular para ingreso, clasificación del riesgo, inicio de tratamiento farmacológico indicado por especialista (Neurólogo) y derivación a Equipo Interdisciplinario de acuerdo a sus necesidades.

- La intervención es un proceso que con enfoque integral e interdisciplinario busca disminuir la incidencia de las complicaciones prevenibles, apoyar e integrar al paciente y a su familia en proceso de tratamiento, disminuir el grado de discapacidad, y optimizar los resultados funcionales y la reintegración social.

Integrantes del Equipo Interdisciplinario:

- Médico: a cargo del tratamiento del paciente es el referente natural para el seguimiento médico y, por lo tanto, debe vincularse precozmente al proyecto terapéutico. Su papel es esencial para la observancia de las medidas de prevención secundarias y para la adaptación de los tratamientos según su eficacia y su tolerancia. El seguimiento también es indispensable desde el punto de vista de la rehabilitación. Según los expertos se recomienda su intervención cada 6 meses. 
- Kinesiólogo: utiliza la enseñanza, los ejercicios y la manipulación física del cuerpo del paciente con la intención de restaurar el movimiento, equilibrio y la coordinación. El objetivo de la terapia física es lograr que el paciente que sufre un ACV, vuelva a aprender actividades motoras simples, tales como caminar, sentarse, ponerse de pie, acostarse y el proceso de cambiar de un tipo de movimiento a otro.

- Fonoaudiólogo: interviene en 2 grandes áreas, la comunicación y la deglución, las cuales frecuentemente se ven afectadas por el ACV. Entrega diversos beneficios, para obtener una rehabilitación satisfactoria y funcional para el paciente y la familia y/o cuidadores.

- Enfermera: función primordial, serían darle al paciente todas las atenciones necesarias que propendan al logro de recuperar su salud y su re-inserción a la comunidad donde vive.

- Psicólogo: asistir directamente al paciente que se encuentra en dificultades relacionadas a su comportamiento o forma de ser, en su mayoría. Este tipo de profesional no soluciona de forma directa los problemas, sino que más bien sirve como canalizador para que el afectado comprenda cuál es el problema que padece y cómo solucionarlo con las herramientas de las que dispone.

- Asistente Social: su rol fundamental a la hora de reforzar una mirada global, estructural y sistémica de la situación de salud y enfermedad del paciente y su familia esto significa aportar a una reflexión permanente en torno a las condiciones estructurales que genera las consecuencia de esta enfermedad; así como la consideración de todos aquellos factores protectores que contribuyen a garantizar la salud y una mejor calidad de vida del paciente.

- Medicina Complementaria (Acupuntura): práctica de la medicina tradicional china y japonesa que consiste en la introducción de agujas muy finas en determinados puntos del cuerpo humano para aliviar dolores, anestesiar determinadas zonas y curar ciertas enfermedades, esta práctica la realiza un médico capacitado en el Centro de Salud Familiar.

- Técnicos Paramédicos: Su función es realizar todas aquellas acciones de salud que le sean solicitadas, de acuerdo a las normas preestablecidas, por el profesional médico enfermera según sea el caso.

3. Intervenciones educativas utilizando la estrategia de "Educación para la Salud":

- Es una herramienta para promocionar la salud consistente en un proceso educativo (charlas y/o talleres) encaminada a que las personas aprendan a modificar sus conductas de riesgo y lograr los cambios ambientales, económicos y sociales que favorezcan las conductas saludables. Se tendrá presente en todas las atenciones y se realizará, de forma individual a partir de la relación personal de los profesionales con los pacientes $\mathrm{y}$, de forma colectiva mediante acciones dirigidas a grupos de población. Y será responsabilidad de todos los miembros del Equipo Interdisciplinario que la desarrollaran tanto durante la actividad cotidiana como en aquellas actividades específicas dentro de cada uno de los programas.

- Educar sobre la modificación de estilos de vida y eliminación de hábitos no saludables, además de transmitir la importancia de controles y adherencia al tratamiento farmacológico.

- Todo paciente con ACV debe recibir educación y consejo en relación con los factores del estilo de vida que pueden reducir su riesgo de recurrencia

4. Taller de Adherencia Farmacológica: Este taller será realizado por el Químico Farmacéutico del Centro de Salud Familiar. La adherencia terapéutica es el grado de coincidencia del comportamiento de un paciente en relación a los medicamentos que debe tomar, el seguimiento de una dieta o los cambios que ha de hacer en su estilo de vida, con las recomendaciones de los profesionales de la salud. Resalta sobretodo la participación activa del paciente en la toma de decisiones, es decir le hace protagonista de su propia salud, resulta imprescindible, por tanto que el paciente asuma el papel de enfermo crónico y ponga en marcha todos los cambios que serán necesarios para lograr un adecuado ajuste a la enfermedad.

5. Visitas Domiciliarias Integrales: Actividad que se realiza por uno o más integrantes del equipo de salud en el domicilio de una familia, con el objetivo de establecer una integración con uno o más miembros y su entorno para conocer su medio ambiente y darles apoyo para enfrentar problemas biopsicosociales, en el marco de una relación asistencial continua e integral, que debe ser la característica del quehacer en la atención primaria. Se podría agregar que tiene un componente centrado en las acciones de fomento, protección, recuperación y/o rehabilitación de la salud

6. Actividades para la Comunidad: A continuación se detallan actividades que se desarrollara en beneficio de la comunidad que tendrá como finalidad dar a conocer los factores de riesgo del ACV, sus cuidados y estilos de vidas saludables:

- Talleres educativos de Promoción de Salud.

- Talleres de actividad física.

- Reuniones interactivas y/o consultivas, con grupos específicos.

- Talleres preventivos en población con factores de riesgo cardiovascular

7. Canasta de Prestaciones: El Centro de Salud Familiar es un establecimiento público, e igual que todas las instituciones gubernamentales cuenta con un presupuesto anual, dividido en tres grupos: por ejemplo para el año 2014 US\$3.628.533 para el pago del Recursos Humanos, US\$1.984.814 para bienes y servicios de Consumo e Inversiones US\$12.365. El presupuesto asignado se financia principalmente $(98 \%)$ de los fondos provenientes de FONASA, y que corresponde a la producción realizada por el establecimiento.

A continuación se presenta el detalle de una canasta de prestaciones para estimar el costo del Plan de Intervención Integral, la base de costos corresponde al año 2014 
Tabla 3: Costo Plan de Intervención Integral

\begin{tabular}{|c|c|c|c|c|c|}
\hline Prestación & $\mathrm{N}^{\mathrm{o}}$ consultas Año & $\begin{array}{l}\text { Duración } \\
\text { Minutos }\end{array}$ & Valor Hora & Costo Atención & Costo Anual \\
\hline Médico & 4 & 30 & 18,5 & 9,3 & 37,1 \\
\hline Nutricionista & 4 & 30 & 8,8 & 4,4 & 17,5 \\
\hline Enfermera & 4 & 30 & 8,8 & 4,4 & 17,5 \\
\hline Kinesiólogo & 12 & 30 & 8,8 & 4,4 & 52,6 \\
\hline Fonoaudiólogo & 12 & 30 & 8,8 & 4,4 & 52,6 \\
\hline Psicólogo & 12 & 30 & 8,8 & 4,4 & 52,6 \\
\hline Asistente Social & 4 & 30 & 8,8 & 4,4 & 17,5 \\
\hline Técnico Paramédico & 3 & 30 & 4,1 & 2,0 & 6,1 \\
\hline Medicina Complementaria (Acupuntura) & 4 & 30 & 9,3 & 4,6 & 18,5 \\
\hline Total & 59 & & & & $272,1^{*}$ \\
\hline
\end{tabular}

El costo anual en relación a las prestaciones otorgadas por profesionales de la Salud corresponde a US\$272,1. Esta canasta de prestaciones se basa en los valores que a la fecha paga el Centro de Salud Familiar por hora a sus profesionales, el valor pagado por hora se calcula en base al promedio de financiamiento que tiene los programas y a la regulación del mercado en relación a cada tipo de profesionales.

Tabla 4: Medicamentos del tratamiento farmacológico, la frecuencia y su costo anual.

\begin{tabular}{|l|c|c|c|c|c|c|}
\hline \multicolumn{1}{|c|}{ Medicamento } & Código & Valor & $\begin{array}{c}\text { Cantidad por } \\
\text { Día }\end{array}$ & $\begin{array}{c}\text { Cantidad por } \\
\text { Mes }\end{array}$ & $\begin{array}{c}\text { Cantidad por } \\
\text { Año }\end{array}$ & $\begin{array}{c}\text { Costo Anual } \\
\text { Aspirina } 100 \mathrm{Mg}\end{array}$ \\
\hline Atrovastatina $20 \mathrm{Mg}$ & $214-0110$ & 0,0031 & 2 & 60 & 720 & 2,23 \\
Losartan $50 \mathrm{Mg}$ & $213-2600$ & 0,0294 & 2 & 60 & 720 & 21,14 \\
\hline Total & & 0,0216 & $1 / 4$ & 7.5 & 90 & 1,95 \\
\hline
\end{tabular}

Los valores de los medicamentos se obtuvieron de la base de datos de compras del Centro de Salud Familiar, estos valores son licitados. Los medicamentos que se observan en la canasta es aquel que se indica post alta hospitalaria a los pacientes con ACV para continuar su tratamiento en la atención primaria.

Es necesario monitorear en forma sistemática y planificada, el grado de cumplimiento del procedimiento intervención oportuna a los pacientes dados de alta por ACV para evaluar si el desempeño cumple con los niveles esperados de calidad de servicio o captar situaciones que se desvían de lo previsto o sean problemáticas y poder efectuar medidas correctoras a tiempo como mejora continua de la calidad de atención.

Se establece un indicador global el cual será monitoreado en forma trimestral por el Jefe del Programa Cardiovascular, el cual servirá para contribuir a la meta de gestión ministerial la cual consiste en que estos pacientes deben ser citados con médico en Atención Primaria de Salud dentro de 60 días desde el alta hospitalaria.

\section{$\mathrm{N}^{\mathrm{o}}$ de pacientes ingresados intervenidos durante el trimestre}

\section{$\mathrm{N}^{\mathrm{o}}$ de pacientes ingresados al Programa Cardiovascular durante el trimestre}

El porcentaje óptimo de cumplimiento para el Indicador de Monitoreo del Plan de Intervención Integral será de 90-100\%, si este no se cumple se implantarán planes de mejora para lograr el objetivo.

Y finalmente la tabla 5 presenta el flujo resumido de derivación y atención al paciente con ACV del Plan de Intervención Integral con 8 grandes etapas. 
Tabla 5: Flujo de derivación y atención al paciente con ACV.

\begin{tabular}{|c|c|c|c|c|}
\hline $\mathbf{N}^{\circ}$ & Unidad & Responsable & Flujo & Descripción \\
\hline 1 & GES & Jefe Unidad & Oficina GES & Recepción de contrarreferencia del hospital \\
\hline 2 & Sector & Coordinador Sector & $\begin{array}{l}\text { Sector inscrito } \mathrm{Pa}- \\
\text { ciente }\end{array}$ & $\begin{array}{l}\text { Verificación y envío de contrarreferencia a } \\
\text { coordinación sector para gestionar cita a mé- } \\
\text { dico }\end{array}$ \\
\hline 3 & $\begin{array}{l}\text { Equipo Programa } \\
\text { Cardiovascular }\end{array}$ & Jefe Programa Cardiovascular & $\begin{array}{l}\text { Oficina Coordinación } \\
\text { Cardiovascular }\end{array}$ & $\begin{array}{l}\text { Recepción y análisis de antecedentes para in- } \\
\text { greso al Programa Cardiovascular }\end{array}$ \\
\hline 4 & Sector & Médico & Consulta & Inicio de tratamiento de acuerdo a Guía Clínica \\
\hline 5 & Farmacia & Jefe de Farmacia & Farmacia Central & $\begin{array}{l}\text { Entrega de medicamentos y orientación según } \\
\text { indicación }\end{array}$ \\
\hline 6 & Sector & Médico & Consulta & $\begin{array}{l}\text { Evaluación daño ACV y deriva a Equipo Inter- } \\
\text { disciplinario }\end{array}$ \\
\hline 7 & Sector & $\begin{array}{l}\text { Equipo Interdisciplinario: } \\
\text { Kinesiólogo, Asistente Social, } \\
\text { Psicólogo, Fonoaudiólogo, } \\
\text { Nutricionista, Enfermera, Quí- } \\
\text { mico Farmacéutico, Técnico } \\
\text { de Salud }\end{array}$ & $\begin{array}{l}\text { Sector inscrito } \mathrm{Pa}- \\
\text { ciente }\end{array}$ & $\begin{array}{l}\text { Evaluación e identificación de factores biomé- } \\
\text { dicos, psicológicos y sociales previos. Aplica- } \\
\text { ción de acciones integrales y terapias Interdis- } \\
\text { ciplinarias. }\end{array}$ \\
\hline 8 & Sector & Jefe Programa Cardiovascular & $\begin{array}{l}\text { Oficina Coordinación } \\
\text { Cardiovascular }\end{array}$ & $\begin{array}{l}\text { Reunión de coordinación con Equipo Salud } \\
\text { Cardiovascular, para seguimiento continuo de } \\
\text { tratamiento de acuerdo a Guía Clínica y Plan } \\
\text { Integral de Atención }\end{array}$ \\
\hline
\end{tabular}

El Plan también establece las unidades, los responsables y el flujo y las acciones que se deben realizar de forma general para lograr una intervención integral.

De acuerdo a los objetivos propuestos y tomando en consideración el estudio realizado, el análisis e interpretación de los datos presentan las conclusiones más relevantes son:

- De acuerdo al estudio realizado se pudo observar que las ECV son la principal causa de muerte en Chile y en la provincia de Nuble.

- El análisis estadísticos de datos permitió caracterizar a la población con ACV del Centro de Salud Familiar, la cual se destaca por ser una población adulta mayor de 65 años y más, rango donde se presenta una mayor cantidad de casos, pero también se observó otro grupo relevante, es el que se encuentra entre los 46 y 64 años que registra un número importante de pacientes con ACV, los que por su edad son considéranos autovalentes por lo tanto son un grupo a los cuales se puede intervenir para prevenir un nuevo ACV, en cuanto al sexo no se presenta como un factor de riesgo ya que no existe diferencia significativa entre ellos.

- Las estrategias del Plan de Intervención Integral tienen un enfoque integral e interdisciplinario el cual busca disminuir la incidencia de las complicaciones prevenibles, apoyar e integrar al paciente y a su familia en proceso de tratamiento, disminuir el grado de discapacidad, $\mathrm{y}$ optimizar los resultados funcionales y la reintegración social.

- Entre un $40 \%$ a un $50 \%$ de las personas que sufren accidentes cerebrales tienen secuelas que van a requerir algún grado de apoyo. Por ello el Plan de Intervención Integral está compuesto por distintos profesionales de la salud para conseguir una situación funcional, familiar y social lo más próxima a la que el paciente tenía previo al accidente cerebrovascular.

\section{Discusión}

Los resultados de este estudio, confirman la situación crítica que presenta el país y la provincia en la prevención, manejo y recuperación de los pacientes con ACV. La atención post hospitalaria que actualmente recibe los pacientes es principalmente farmacológica, y su seguimiento es realizado a través de controles que no siempre permiten evaluar adecuadamente la situación integral del paciente, si bien las orientaciones o Guías Clínicas sugieren manejar al paciente de forma integral, en la práctica no ha sido implementado. Sin embargo se requiere abordar este problemática de salud de forma prioritaria, para lo cual esta investigación elabora una propuesta factible de implementar. Se espera que los resultados de este estudio aporten información relevante para lograr una comprensión más acabada del problema desde la perspectiva nacional y local y sirva para mejorar el diseño y la implementación de estrategias de salud pública.

Este estudio destaca la importancia de contar con un Equipo Interdisciplinario capacitado en el manejo integral de pacientes con ACV desde la atención primaria, otorgándoles herramientas para una adecuada intervención, y particularmente con la mirada global de este problema de salud.

La formación de los integrantes de los Equipos Interdisciplinarios debe perfeccionarse y actualizarse permanentemente, de acuerdo a las necesidades 
de los pacientes con ACV, para lo cual resultará útil encomendar su revisión continua a las entidades científicas y formadoras de recurso humano en salud.

Dada la importancia de este problema de salud presenta para el país y la provincia, se requiere implementar intervenciones sanitarias, y estudiar el impacto que estas tendrán, es importante conocer desde la perspectiva del equipo de salud y desde la perspectiva de las pacientes y familia, la relación con los factores que facilitan o dificultan la continuidad del proceso de atención.

La investigación ofrecería información para mejorar los resultados, la cual además puede replicarse en otras regiones geográficas para obtener información homogénea, como diagnóstico inicial de un plan de salud de primer nivel con proyección nacional.

Agradecimientos: La presente investigación es producto de un Seminario de Titulación para optar al Título de Ingeniero en Administración de Empresas de la Facultad de Ciencias Empresariales de la Universidad del Bio Bio.

Especial agradecimiento a la Directora Dra. Nancy Jara Jara y al Equipo de Salud del Programa Cardiovascular del Centro de Salud Familiar Violeta Parra, Chillán, Chile, y a los directivos y referentes técnicos de la Dirección del Servicio de Salud Ñuble, Chillán, Chile, por las facilidades otorgadas para la presente investigación.

Conflictos de intereses: Los autores declaramos que no presentamos conflictos de intereses.

\section{Referencias}

1. Organización Panamericana de la Salud (OPS) de la Organización Mundial de Salud (OMS), 2017.

2. Organización Panamericana de la Salud (OPS): Salud en las Américas, 2007.

3. Ministerio de Salud (MINSAL). Departamento de Estadísticas e Información de Salud (DEIS). Indicadores Básicos de Salud Chile 2013, 2013.

4. Ministerio de Salud (MINSAL). Estrategia Nacional de Salud para el Cumplimiento de los Objetivos Sanitarios de la Década 2011-2020, 2011.

5. Icaza G, Núñez L. Atlas de Mortalidad Cardiovascular. PIFRECV, Universidad de Talca, 2006

6. Ministerio de Salud (MINSAL). Estrategia Nacional de Salud para el Cumplimiento de los Objetivos Sanitarios de la Década 2011-2020

7. Lavados P, Sacks C, Prina L, Escobar A, Tossi C, Araya F. Incidence, 30-days case-fatality rate, and prognosis of stroke in Iquique, Chile; a 2-year community-based prospective study (PISCIS Project). Lancet 365: 2206-2215, 2005.

8. Nacional Institute of Neurological Disorders and Stroke (NINDS). Accidente cerebrovascular: Esperanza en la investigación. NIH 01-2222s. 2007.

9. WHO. The Atlas of Heart Disease and Stroke. 2004

10. Ministerio de Salud (MINSAL). Secretaria Ministerial de Salud (SEREMI). Diagnóstico Región del Biobío con enfoque en Determinantes Sociales de la Salud, 2013.

11. Escobar MC. Prevención del riesgo cardiovascular: políticas chilenas Chilean Policy. Rev. Med. Clin. Condes. 2012; 23(6) 651-655.

12. Ministerio de Salud. Programa Salud Cardiovascular, Reorientación de los Programas de Hipertensión y Diabetes,
División de Rectoría y Regulación Sanitaria, Departamento de Programas de las Personas, Programa Salud del Adulto, 2002.

13. Ministerio de Salud (MINSAL). Implementación del Enfoque de Riesgo en el Programa Salud Cardiovascular, 2009.

14. Huerta P, Paul I, Leyton C. Impacto de Indicadores de Gestión en Salud sobre Estrategias de un Servicio de Salud Público. Revista de Salud Pública de Colombia, 2012; 14(2), 248-259. 